\title{
Hidratação enteral em equinos - solução eletrolítica associada ou não à glicose, à maltodextrina e ao sulfato de magnésio: resultados de laboratório
}

\author{
Enteral fluid therapy in horses - electrolyte solution associated or not with glucose, maltodextrine \\ and magnesium sulphate: laboratory results
}

\author{
Marcel Ferreira Bastos Avanza ${ }^{\mathrm{I}}$ José Dantas Ribeiro Filho $^{\mathrm{I} *}$ Marco Aurélio Ferreira Lopes $^{\mathrm{I}}$ \\ Fernanda Saules Ignácio ${ }^{I}$ Thony Assis CarvalhoI José Domingos Guimarães ${ }^{I}$
}

\section{RESUMO}

No presente estudo, foram avaliados os efeitos de soluções eletrolíticas administradas via sonda nasoesofágica de pequeno calibre sobre o hematócrito, o volume plasmático e as concentrações plasmáticas de proteínas totais, sódio, potássio, cloreto, magnésio total e cálcio iônico de eqüinos hígidos e desidratados experimentalmente. Foram utilizados quatro equinos adultos, mestiços, dois machos e duas fêmeas. No experimento 1 (E1Des), os animais foram desidratados experimentalmente, enquanto no 2 (E2Hig) foram utilizados equinos hígidos. Os animais foram submetidos a cada um dos seguintes tratamentos: SE - solução eletrolítica isotônica; SEGli - solução eletrolítica isotônica + glicose; SEMalt - solução eletrolítica isotônica + maltodextrina e SEMg - solução eletrolítica isotônica + sulfato de magnésio. A solução eletrolítica foi administrada na dose de $15 \mathrm{~mL} \mathrm{~kg}^{-1} \mathrm{~h}^{-1}$, durante $12 \mathrm{~h}$ via sonda nasoesofágica por fluxo contínuo. Os tratamentos com as soluções eletrolíticas ocasionaram expansão do volume plasmático, ocasionando a redução nos valores das proteínas plasmáticas totais e do hematócrito, enquanto os valores dos eletrólitos avaliados permaneceram na faixa de referência.

Palavras-chave: equinos, hidratação, eletrólitos, glicose, maltodextrina, sulfato de magnésio.

\section{ABSTRACT}

In the present study, the effects of four different electrolyte solutions on the packed cell volume (PCV), plasma volume and plasma concentrations of total protein, sodium, potassium, chloride, total magnesium, and ionized calcium in healthy and experimentally dehydrated horses were evaluated. Four crossbred horses, two males and two females were used. In experiment 1 (E1Des) the animals were experimentally dehydrated, while in the second experiment (E2Hig) healthy equines were used. In both experiments the animals were subjected to the following treatments: SE - isotonic electrolyte solution; SEGlu - glucose-enriched SE; SEMalt maltodextrine-enriched SE and SEMg - magnesium sulphateenriched SE. The electrolyte solutions used in all treatments were administered at $15 \mathrm{~mL} \mathrm{~kg}^{-1} \mathrm{~h}^{-1}$ during $12 \mathrm{~h}$ through smallbore nasoesophagus tube by continuous flow. The treatments with the electrolyte solutions resulted in an increase of plasma volume and a decrease in total plasma protein, hematocrit, while the electrolytes remained within the reference values.

Key words: horse, fluid therapy, electrolytes, glucose, maltodextrine, magnesium sulfate.

\section{INTRODUÇÃO}

A hidratação é um importante recurso terapêutico utilizado diariamente na rotina clínica. Isso ocorre porque a desidratação usualmente acompanha a maior parte das doenças e síndromes que acometem os equinos, como, por exemplo, sudorese excessiva, diarréia, peritonite difusa, choques endotoxêmico e septicêmico, ectopias do intestino grosso, obstruções intestinais, íleo e cólica, dentre outras (RADOSTITS et al., 2000). A administração de soluções eletrolíticas estéreis por via intravenosa é o método padrão recomendado na literatura (SEAHORN \& CORNICKSEAHORN, 1994). Essa via permite a infusão rápida do volume de reposição, ocasionando a expansão veloz do volume plasmático, por isso o seu uso é indispensável nos casos de desidratação intensa e de choque hipovolêmico. Apesar de sua indiscutível

'Departamento de Veterinária (DVT), Universidade Federal de Viçosa (UFV). Av. P. H. Rolfs s/n, Centro, 36570-000, Viçosa, MG,

Brasil. E-mail: dantas@ufv.br. *Autor para correspondência. 
eficácia, a hidratação por via intravenosa, algumas vezes, mostra-se de custo elevado, isso porque, para muitas condições clínicas em eqüinos, grandes volumes de fluidos precisam ser administrados (AVANZA, 2007).

Uma alternativa para a administração de fluidos é a via nasoesofágica ou nasogástrica. Com o emprego de sonda de pequeno calibre, a via enteral é uma forma fisiologicamente segura para se administrar fluidos, pois a mucosa do trato gastrintestinal atua como uma barreira seletiva natural para a absorção, não exigindo soluções estéreis e composição finamente ajustada (LOPES, 2002). Por isso, a hidratação enteral (HET) é uma alternativa de reidratação eficiente e econômica quando o paciente necessita de grandes volumes de solução eletrolítica. O uso dessa técnica, empregada com sucesso em equinos e bovinos, permite que os animais sejam mantidos em baias sem a necessidade de contenção física enquanto a hidratação é realizada continuamente, sendo seguro permitir o acesso a alimentos. Além disso, não há necessidade de reintroduções freqüentes da sonda, o que reduz o risco de lesões no esôfago e na faringe e diminui o número de vezes em que o animal precisa ser contido, minimizando o estresse devido ao tratamento (AVANZA et al., 2004; RIBEIRO FILHO et al., 2004). A HET é eficiente na recomposição da homeostase por aumentar o volume plasmático, restabelecer a concentração sérica de eletrólitos, o pH sanguíneo, a viscosidade da digesta, além de promover a diurese (LOPES et al., 2002; RIBEIRO FILHO et al., 2007).

Em outras espécies, a adição de glicose à solução para HET maximiza a absorção de água (GISOLFI \& DUCHAN, 1992). Entretanto, os resultados de estudos realizados em equinos com soluções contendo glicose não foram satisfatórios (MONREAL et al., 1999). Talvez a substituição da glicose por um polímero de glicose como a maltodextrina nas soluções eletrolíticas para HET possa ocasionar a expansão do volume plasmático de maneira mais eficiente nos animais dessa espécie. Por isso, um grande desafio na correção dos desequilíbrios hidroeletrolíticos e ácidosbase em eqüinos e ruminantes utilizando-se a hidratação por via enteral é a elaboração de protocolos de tratamentos práticos, eficazes e econômicos que possam recompor e manter a homeostase desses animais. O objetivo do presente estudo foi verificar o efeito de solução eletrolítica associada ou não à glicose, à maltodextrina e ao sulfato de magnésio sobre o hematócrito, o volume plasmático e as concentrações plasmáticas de proteínas totais, sódio, potássio, cloreto, magnésio total e cálcio iônico em equinos hígidos e desidratados experimentalmente.

\section{MATERIAL E MÉTODOS}

Foram utilizados quatro eqüinos mestiços, dois machos castrados e duas fêmeas, hígidos, com uma fístula no cólon dorsal direito, com idade entre sete e 14 anos, escore corporal de três a quatro e peso corporal entre 285 e $391 \mathrm{~kg}$. Trinta dias antes do experimento e durante a fase experimental, os animais foram mantidos em baias individuais. Receberam feno de capim tifton 85, suplemento mineral e água ad libitun. $\mathrm{O}$ estudo foi dividido em dois experimentos. No experimento 1 (E1Des), os animais foram desidratados experimentalmente por meio de jejum hídrico e alimentar de 24 horas e aplicação de uma única dose de $3 \mathrm{mg} \mathrm{kg}^{-1}$ de furosemida por via intravenosa, esperando-se uma desidratação discreta a moderada. No experimento 2 (E2Hig), foram utilizados eqüinos hígidos. Antes do início de cada período de tratamento foi introduzida em cada animal uma sonda nasoesofágica ${ }^{\mathrm{a}}$ de $6 \mathrm{~mm}$ de diâmetro e $130 \mathrm{~cm}$ de comprimento fixada ao cabresto, para a administração das soluções. Em seguida, iniciaram-se os tratamentos: grupo SE - a solução foi preparada utilizando-se 5,27g de $\mathrm{NaCl}, 0,37 \mathrm{~g}$ de $\mathrm{KCl}$ e 3,78g de $\mathrm{NaHCO}_{3}$ em $1.000 \mathrm{~mL}$ de água (osmolaridade calculada: 280mosmol L-1 ); grupo SEGli - a solução foi preparada adicionando 10,8g de glicose em um litro da solução eletrolítica (osmolaridade calculada: 340mosmol L ${ }^{-1}$ ); grupo SEMalt - a solução foi preparada adicionando $18 \mathrm{~g}$ de maltodextrina ${ }^{\mathrm{b}}$ em um litro da solução eletrolítica (osmolaridade calculada: 290mosmol L-1) e grupo SEMg - a administração da solução eletrolítica foi precedida pela administração de uma dose de sulfato de magnésio $\left(1 \mathrm{~g} \mathrm{~kg}^{-1}\right)$ diluído nos primeiros cinco litros da solução eletrolítica. $\mathrm{O}$ volume das soluções administradas nos animais de todos os tratamentos foi de $15 \mathrm{~mL} \mathrm{~kg}^{-1} \mathrm{~h}^{-1}$, durante $12 \mathrm{~h}$ via sonda nasoesofágica por fluxo contínuo. Durante a hidratação o acesso à água foi suspenso, entretanto o acesso ao feno e suplemento mineral foi mantido.

Os tempos do experimento E1Des tiveram a seguinte distribuição: T-24h (imediatamente antes do início da desidratação), T0h (final do período de desidratação), T6h (seis horas de hidratação), T12h (12 horas de hidratação), T18h e T24h (seis e 12 horas após o término da hidratação, correspondente à fase de avaliação final). Enquanto os tempos do experimento E2Hig foram: T0h (imediatamente antes do início da hidratação), T6h (seis horas de hidratação), T12h (12 horas de hidratação), T18h (seis horas após o término da hidratação) e T24h (12 horas após o término da hidratação).

A coleta de sangue foi realizada após antissepsia, por venipunção jugular, utilizando-se 
agulhas e frascos Vacutainer ${ }^{c}$ contendo EDTA para mensuração do hematócrito e das proteínas plasmáticas totais. O hematócrito foi determinado pela técnica do microhematócrito, e a concentração de proteínas plasmáticas totais foi determinada pelo método da refratometria (JAIN, 1986). Alíquotas de plasma foram obtidas após a centrifugação (3396G por cinco minutos) de amostras de sangue coletadas em frascos Vacutainer contendo heparina de lítio para a determinação das concentrações de sódio e potássio, os quais foram dosados por fotometria de chama (Celm) ${ }^{\mathrm{d}}$; cloreto (método Colorimétrico) e magnésio total (método Mane Yoe) e analisados com auxílio de reagentes bioclin e leitura em equipamento automático ${ }^{\text {e }}$ o cálcio ionizado foi realizado com auxílio de reagentes Iselab e leitura em seletor de íons ${ }^{f}$. O volume plasmático ${ }^{g}$ foi determinado pelo corante azul de Evans, segundo metologia descrita por AVANZA (2007).

Todas as variáveis estudadas foram submetidas à análise de variância para verificar os efeitos do tratamento e do tempo. Para tanto, foi utilizado o delineamento em quadrado latino $4 \times 4$, considerando os efeitos de período, animal, tratamento e tempo. Este foi empregado sob o enfoque da metodologia dos modelos mistos em medidas repetidas, considerando o efeito de animal como aleatório. As análises foram realizadas no programa estatístico SAEG (SAEG-UFV, 9.1,2007). Quando houve significância pelo teste $F$, as médias foram comparadas pelo teste Tukey $(\mathrm{P}<0,05)$. As análises não-paramétricas (Kruskal-Wallis) foram efetuadas para dados que não apresentaram distribuição normal ou homogeneicidade das variâncias.

\section{RESULTADOS E DISCUSSÃO}

No presente estudo, nos animais do E1Des na fase de indução da desidratação (T-24h), foi observado pequeno aumento nos valores do hematócrito, decorrente do jejum hídrico associado à administração da furosemida (Tabela 1). Diferença significativa no aumento do hematócrito foi obtida por RIBEIROFILHO \& ALVES (2002) eALVES et al.(2005), os quais desidrataram equinos experimentalmente. No E1Des, a desidratação discreta foi confirmada pelo aumento da PPT. Contudo, apenas os equinos do SEMg exibiram aumento significativo $(\mathrm{P}<0,005)$. Esse achado possivelmente deveu-se à variação individual, pois o protocolo de desidratação foi semelhante para os animais de todos os tratamentos. Durante a fase de hidratação (T6h e T12h), em todos os tratamentos do E1Des e E2Hig, foi constatada diminuição nos valores do hematócrito, demonstrando a eficácia da hidratação enteral (HET) na expansão do volume plasmático (Tabela 1 e 2), corroborando os achados de LOPES et al. (2002) e ALVES et al. (2005). Entretanto, no presente ensaio, o aumento (T-24h) e o decréscimo (T6h e T12h) nos valores do hematócrito não foram significativos $(\mathrm{P}>0,05)$. Esse achado sugere que provavelmente $\mathrm{o}$ período de desidratação (24 horas), o tempo de hidratação (12 horas) e o volume das soluções (15mL $\mathrm{kg}^{-1}$ ) foram insuficientes.

No E1Des, após a administração da HET (T6h e T12h), foi observada também redução significativa da PPT $(\mathrm{P}<0,05)$, exceto para o tratamento $\mathrm{SE}(\mathrm{P}>0,05)$. Esse resultado demonstrou que a PPT foi eficiente na expansão da volemia (Tabela 1 e 2), assemelhando-se aos resultados obtidos por LOPES et al. (2002) e ALVES et al. (2005). Nos animais do E2Hig (Tabela 2), os valores das PPT dos tratamentos SEMalt e SEGli não apresentaram diminuição significativa $(\mathrm{P}>0,05)$ durante a HET (6h e 12h). Esse achado corrobora os resultados de SOSA LEON et al. (1995) e MONREAL et al. (1999), os quais não encontraram diminuição significativa do volume plasmático nos animais que receberam soluções que continham glicose.

No E1Des, o sódio plasmático não apresentou diferença entre tratamentos $(\mathrm{P}>0,05)$. Entretanto, houve diminuição $(\mathrm{P}<0,05)$ gradual a partir do término da hidratação (12h) no SE e SEGli até o final do período de observação (T24h) e no SEMalt no período de observação (T18h e T24h), em consequência da correção da desidratação dos pacientes através da HET e da ingestão espontânea de água na fase de observação (Tabela 1), corroborando os achados de ALVES et al. (2005). No E2Hig foi observado aumento $(\mathrm{P}<0,05)$ do sódio plasmático nos tratamentos SE e SEMg durante o período final de hidratação (T12h). Possivelmente a ausência de glicose e maltodextrina nas soluções SE e SEMg pode ter contribuído para esse evento, pois, conforme SOSA LEON et al. (1995) e MONREAL et al. (1999) citaram, para ocorrer a absorção de sódio, é necessário que ele esteja combinado à glicose, conseqüentemente, nessas duas soluções o seu valor foi superior às soluções SEGli e SEMalt. No entanto, não foi observada hipernatremia, pois as pequenas variações observadas encontravam-se na faixa de referência (KANEKO et al., 1997). Ainda nos animais dos E2Hig, tratamentos SE e SEMalt no T24h, houve redução significativa $(\mathrm{P}<0,05)$ nos valores de sódio possivelmente ocasionada por variação individual (Tabela 2). Assim como no E2Hig, as pequenas variações observadas no E1Des situaramse na faixa de referência, que, segundo KANEKO et al. (1997), é de 132-146mmol L-1. 
Tabela 1 - Hematócrito, proteínas plasmáticas totais, sódio, potássio, cloreto, magnésio total, cálcio iônico e volume plasmático em eqüinos desidratados submetidos à hidratação enteral com solução eletrolítica (SE) associada ou não à glicose (SEGli), à maltodextrina (SEMalt) e são ulfato de magnésio (SEMg).

\begin{tabular}{|c|c|c|c|c|c|c|}
\hline Tratamento & $\mathrm{T}-24 \mathrm{~h}$ & T0h & T6h & $\mathrm{T} 12 \mathrm{~h}$ & $\mathrm{~T} 18 \mathrm{~h}$ & T24h \\
\hline \multicolumn{7}{|c|}{---------------------------------------------------------Hematócrito \% (médiaさdesvio padrão) ------------------------------------------------------- } \\
\hline SE & $30,0 \pm 4,1 \mathrm{Aa}$ & $32,7 \pm 4,5 \mathrm{Aa}$ & $29,5 \pm 4,6 \mathrm{Aa}$ & $28,5 \pm 4,5 \mathrm{Aa}$ & $29,0 \pm 5,0 \mathrm{Aa}$ & $30,5 \pm 5,3 \mathrm{Aa}$ \\
\hline SEGli & $27,2 \pm 3,9 \mathrm{Aa}$ & $32,0 \pm 1,6 \mathrm{Aa}$ & $29,0 \pm 3,7 \mathrm{Aa}$ & $28,2 \pm 2,4 \mathrm{Aa}$ & $28,5 \pm 3,1 \mathrm{Aa}$ & $28,7 \pm 2,1 \mathrm{Aa}$ \\
\hline SEMalt & $30,2 \pm 1,7 \mathrm{Aa}$ & $32,2 \pm 2,6 \mathrm{Aa}$ & $29,5 \pm 3,0 \mathrm{Aa}$ & $28,7 \pm 2,9 \mathrm{Aa}$ & $28,7 \pm 4,3 \mathrm{Aa}$ & $28,7 \pm 3,0 \mathrm{Aa}$ \\
\hline SEMg & $30,5 \pm 2,9 \mathrm{Aa}$ & $32,2 \pm 2,2 \mathrm{Aa}$ & $29,5 \pm 2,9 \mathrm{Aa}$ & $27,7 \pm 2,2 \mathrm{Aa}$ & $28,0 \pm 0,8 \mathrm{Aa}$ & $27,5 \pm 1,0 \mathrm{Aa}$ \\
\hline \multicolumn{7}{|c|}{ 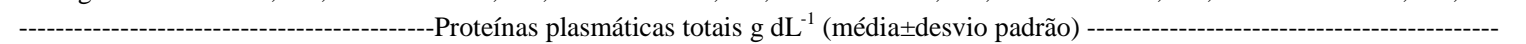 } \\
\hline SE & 7,5 $\pm 0,7 \mathrm{Aa}$ & $7,9 \pm 0,4 \mathrm{Aa}$ & $7,2 \pm 0,5 \mathrm{Aa}$ & $7,0 \pm 0,5 \mathrm{Aa}$ & $7,2 \pm 06 \mathrm{Aa}$ & $7,4 \pm 0,4 \mathrm{Aa}$ \\
\hline SEGli & $7,4 \pm 0,3 \mathrm{ABa}$ & $7,9 \pm 0,3 \mathrm{Aa}$ & $7,2 \pm 0,2 \mathrm{ABa}$ & $6,9 \pm 0,2 \mathrm{Ba}$ & $7,1 \pm 0,3 \mathrm{Ba}$ & $7,2 \pm 0,4 \mathrm{Ba}$ \\
\hline SEMalt & $7,2 \pm 0,6 \mathrm{ABa}$ & $8,0 \pm 0,3 \mathrm{Aa}$ & $7,2 \pm 0,4 \mathrm{ABa}$ & $7,0 \pm 0,3 \mathrm{Ba}$ & $7,2 \pm 0,4 \mathrm{ABa}$ & $7,3 \pm 0,3 \mathrm{ABa}$ \\
\hline SEMg & $7,3 \pm 0,3 \mathrm{Ba}$ & $7,9 \pm 0,1 \mathrm{Aa}$ & $7,3 \pm 0,1 \mathrm{Ba}$ & $7,0 \pm 0,1 \mathrm{Ba}$ & $7,2 \pm 0,4 \mathrm{Ba}$ & $7,3 \pm 0,1 \mathrm{Ba}$ \\
\hline \multicolumn{7}{|c|}{ 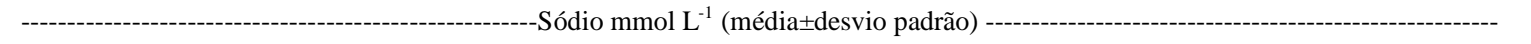 } \\
\hline $\mathrm{SE}$ & & $141,2 \pm 1,0 \mathrm{ABa}$ & $141,7 \pm 1,0 \mathrm{Aa}$ & $139,2 \pm 1,0 \mathrm{Ba}$ & $135,5 \pm 1,3 \mathrm{Ca}$ & $133,2 \pm 1,3 \mathrm{Ca}$ \\
\hline SEGli & & $143,0 \pm 0,8 \mathrm{Aa}$ & $142,7 \pm 2,2 \mathrm{Aa}$ & $139,5 \pm 0,6 \mathrm{Ba}$ & $136,2 \pm 1,0 \mathrm{Ca}$ & $135,2 \pm 1,9 \mathrm{Ca}$ \\
\hline SEMalt & & $142,0 \pm 0,8 \mathrm{Aa}$ & $141,5 \pm 1,3 \mathrm{Aa}$ & $138,7 \pm 1,7 \mathrm{ABa}$ & $136,2 \pm 1,5 \mathrm{BCa}$ & $134,2 \pm 2,1 \mathrm{Ca}$ \\
\hline SEMg & & $140,7 \pm 5,2 \mathrm{Aa}$ & $141,7 \pm 4,6 \mathrm{Aa}$ & 139,5 $\pm 3,1 \mathrm{Aa}$ & $135,7 \pm 3,1 \mathrm{Aa}$ & $134,7 \pm 4,3 \mathrm{Aa}$ \\
\hline \multicolumn{7}{|c|}{ 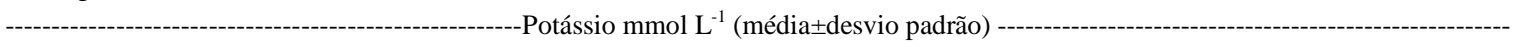 } \\
\hline SE & & 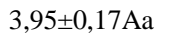 & $3,72 \pm 0,21 \mathrm{Aa}$ & $3,42 \pm 0,51 \mathrm{Aa}$ & $3,52 \pm 0,59 \mathrm{Aa}$ & $4,20 \pm 0,35 \mathrm{Aa}$ \\
\hline SEGli & & $3,80 \pm 0,39$ Аа & $3,35 \pm 0,58 \mathrm{Aab}$ & $3,30 \pm 0,34 \mathrm{Aa}$ & $3,57 \pm 0,19$ Аа & $3,72 \pm 0,48 \mathrm{Aa}$ \\
\hline SEMalt & & $3,95 \pm 0,19$ Аа & $3,22 \pm 0,27 \mathrm{Ab}$ & $3,12 \pm 0,34 \mathrm{Aa}$ & $3,55 \pm 0,62 \mathrm{Aa}$ & $3,92 \pm 0,32 \mathrm{Aa}$ \\
\hline SEMg & & $3,82 \pm 0,21 \mathrm{Aa}$ & 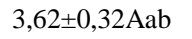 & $3,17 \pm 0,40 \mathrm{Aa}$ & $3,47 \pm 0,44 \mathrm{Aa}$ & $3,80 \pm 0,57 \mathrm{Aa}$ \\
\hline \multicolumn{7}{|c|}{ 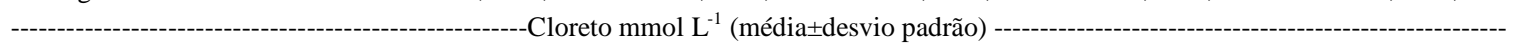 } \\
\hline SE & & $105,5 \pm 4,1 \mathrm{ABa}$ & $108,5 \pm 3,0 \mathrm{Aa}$ & $104,0 \pm 2,2 \mathrm{ABCa}$ & $100,2 \pm 2,2 \mathrm{BCa}$ & $98,7 \pm 1,3 \mathrm{Ca}$ \\
\hline SEGli & & $107,2 \pm 3,4 \mathrm{Aa}$ & $108,2 \pm 3,5$ Аа & $104,2 \pm 2,1 \mathrm{ABa}$ & $100,7 \pm 1,5 \mathrm{Ba}$ & $100,5 \pm 0,6 \mathrm{Ba}$ \\
\hline SEMalt & & $108,0 \pm 2,6 \mathrm{Aa}$ & $109,0 \pm 0,8 \mathrm{Aa}$ & $104,5 \pm 2,4 \mathrm{ABa}$ & $101,5 \pm 2,4 \mathrm{Ba}$ & $100,0 \pm 3,4 \mathrm{Ba}$ \\
\hline SEMg & & $106,7 \pm 2,6 \mathrm{ABa}$ & $109,0 \pm 4,7 \mathrm{Aa}$ & $105,0 \pm 3,6 \mathrm{ABa}$ & $100,0 \pm 3,6 \mathrm{Ba}$ & $99,2 \pm 3,6 \mathrm{Ba}$ \\
\hline \multicolumn{7}{|c|}{ - } \\
\hline $\mathrm{SE}$ & & $2,20 \pm 0,82 \mathrm{Aa}$ & $1,92 \pm 0,05 B C a b$ & $1,75 \pm 0,13 \mathrm{Ca}$ & 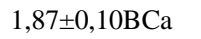 & $1,97 \pm 0,10 \mathrm{Ba}$ \\
\hline SEGli & & $2,15 \pm 0,06 \mathrm{Aa}$ & $1,70 \pm 0,18 \mathrm{BCb}$ & $1,62 \pm 0,15 \mathrm{Ca}$ & $1,95 \pm 0,06 \mathrm{ABa}$ & $2,02 \pm 0,13 \mathrm{Aa}$ \\
\hline SEMalt & & $2,22 \pm 0,12 \mathrm{Aa}$ & $1,67 \pm 0,15 \mathrm{BCb}$ & $1,50 \pm 0,22 \mathrm{Ca}$ & $2,00 \pm 0,10 \mathrm{Aa}$ & $1,97 \pm 0,10 \mathrm{ABa}$ \\
\hline SEMg & & $2,15 \pm 0,58 \mathrm{ABa}$ & $2,22 \pm 0,17 \mathrm{Aa}$ & $1,77 \pm 0,17 \mathrm{Ca}$ & $1,90 \pm 0,14 \mathrm{BCa}$ & $2,02 \pm 0,15 \mathrm{ABCa}$ \\
\hline \multicolumn{7}{|c|}{ 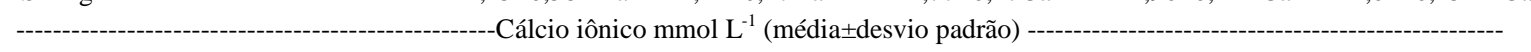 } \\
\hline SE & & $1,58 \pm 0,06 \mathrm{Aa}$ & $1,63 \pm 0,03 \mathrm{Aa}$ & $1,60 \pm 0,13 \mathrm{Aa}$ & $1,67 \pm 0,07 \mathrm{Aa}$ & $1,68 \pm 0,03 \mathrm{Aa}$ \\
\hline SEGli & & $1,61 \pm 0,09 \mathrm{Aa}$ & $1,54 \pm 0,15 \mathrm{Aa}$ & $1,57 \pm 0,24 \mathrm{Aa}$ & $1,68 \pm 0,08 \mathrm{Aa}$ & $1,67 \pm 0,06 \mathrm{Aa}$ \\
\hline SEMalt & & $1,64 \pm 0,02 \mathrm{Aa}$ & $1,47 \pm 0,10 \mathrm{ABa}$ & $1,41 \pm 0,17 \mathrm{Ba}$ & $1,67 \pm 0,04 \mathrm{Aa}$ & $1,64 \pm 0,03 \mathrm{Aa}$ \\
\hline SEMg & & $1,56 \pm 0,07 \mathrm{Aa}$ & $1,56 \pm 0,12 \mathrm{Aa}$ & $1,48 \pm 0,15 \mathrm{Aa}$ & $1,56 \pm 0,18 \mathrm{Aa}$ & $1,62 \pm 0,15 \mathrm{Aa}$ \\
\hline \multicolumn{7}{|c|}{ - } \\
\hline $\mathrm{SE}$ & & $10,02 \pm 1,19$ Аа & $11,82 \pm 1,26 \mathrm{Aa}$ & $14,42 \pm 3,58 \mathrm{Aa}$ & 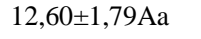 & $11,75 \pm 1,46 \mathrm{Aa}$ \\
\hline SEGli & & $9,80 \pm 1,06 \mathrm{Ba}$ & $13,00 \pm 2,18 \mathrm{ABa}$ & $15,04 \pm 1,85 \mathrm{Aa}$ & $11,54 \pm 2,26 \mathrm{ABa}$ & $9,78 \pm 1,55 \mathrm{Ba}$ \\
\hline SEMalt & & $7,99 \pm 1,21 \mathrm{Ba}$ & $11,97 \pm 1,70 \mathrm{Aa}$ & $14,47 \pm 1,92 \mathrm{Aa}$ & $12,16 \pm 1,37 \mathrm{Aa}$ & $11,44 \pm 1,28 \mathrm{Aa}$ \\
\hline SEMg & & $9,24 \pm 0,74 \mathrm{ABa}$ & $10,81 \pm 1,42 \mathrm{ABa}$ & $14,64 \pm 2,62 \mathrm{Aa}$ & $9,73 \pm 4,25 \mathrm{ABa}$ & $9,05 \pm 1,83 \mathrm{Ba}$ \\
\hline
\end{tabular}

Análise de variância (medidas repetidas)

As médias na mesma coluna seguidas por letras minúsculas diferentes e as médias na mesma linha seguidas por letras maiúsculas diferentes diferem pelo teste de Turkey $(\mathrm{P}<0,05)$.

T-24h (imediatamente antes do início da desidratação), T0h (final do período de desidratação), T6h (seis horas de hidratação), T12h (12 horas de hidratação), T18h e T24h (seis e 12 horas após o término da hidratação, correspondente à fase de avaliação final).

Grupo SE - a solução foi preparada utilizando-se 5,27g de $\mathrm{NaCl}, 0,37 \mathrm{~g}$ de $\mathrm{KCl}$ e 3,78g de $\mathrm{NaHCO}_{3}$ em 1.000 mL de água (osmolaridade calculada: 280mosmol L ${ }^{-1}$ ); grupo SEGli - a solução foi preparada adicionando 10,8g de glicose em um litro da solução eletrolítica (osmolaridade calculada: 340mosmol L ${ }^{-1}$ ); grupo SEMalt - a solução foi preparada adicionando $18 \mathrm{~g}$ de maltodextrina em um litro da solução eletrolítica (osmolaridade calculada: 290mosmol $\mathrm{L}^{-1}$ ) e grupo SEMg - a administração da solução eletrolítica foi precedida pela administração de uma dose de sulfato de magnésio $\left(1 \mathrm{~g} \mathrm{~kg}^{-1}\right)$ diluído nos primeiros cinco litros da solução eletrolítica. 
Tabela 2 - Hematócrito, proteínas plasmáticas totais, sódio, potássio, cloreto, magnésio total, cálcio iônico e volume plasmático em equinos hígidos submetidos à hidratação enteral com solução eletrolítica (SE) associada ou não à glicose (SEGli), à maltodextrina (SEMalt) e ao sulfato de magnésio (SEMg).

\begin{tabular}{|c|c|c|c|c|c|}
\hline Tratamento & T0h & T6h & T12h & T18h & T24h \\
\hline \multicolumn{6}{|c|}{ 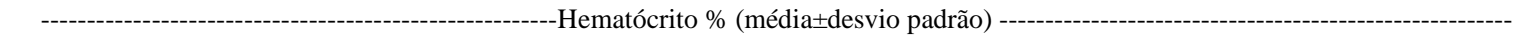 } \\
\hline SE & $31,25 \pm 4,27 \mathrm{Aa}$ & $28,75 \pm 4,03 \mathrm{Aa}$ & $26,50 \pm 3,70 \mathrm{Aa}$ & $25,50 \pm 4,36$ Aа & $31,00 \pm 4,83 \mathrm{Aa}$ \\
\hline SEGli & $30,75 \pm 3,69 \mathrm{Aa}$ & $28,50 \pm 3,52 \mathrm{Aa}$ & $28,25 \pm 3,20 \mathrm{Aa}$ & $29,75 \pm 3,40 \mathrm{Aa}$ & $29,25 \pm 3,10 \mathrm{Aa}$ \\
\hline SEMalt & $31,25 \pm 3,59 \mathrm{Aa}$ & $28,50 \pm 2,52 \mathrm{Aa}$ & $28,50 \pm 1,73 \mathrm{Aa}$ & $27,75 \pm 4,03 \mathrm{Aa}$ & $29,50 \pm 3,79$ Аа \\
\hline \multirow[t]{2}{*}{ SEMg } & $31,00 \pm 5,89 \mathrm{Aa}$ & $27,50 \pm 4,20 \mathrm{Aa}$ & $27,75 \pm 4,27 \mathrm{Aa}$ & $28,75 \pm 3,40 \mathrm{Aa}$ & $29,00 \pm 4,55 \mathrm{Aa}$ \\
\hline & ---------------Pro & plasmáticas totais & ${ }^{-1}$ (média \pm desvio & 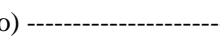 & 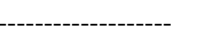 \\
\hline SE & $7,2 \pm 0,4 \mathrm{ABa}$ & $6,7 \pm 0,3 \mathrm{BCa}$ & $6,6 \pm 0,2 \mathrm{BCa}$ & $7,0 \pm 0,3 \mathrm{ABCa}$ & 7,5 $\pm 0,2 \mathrm{Aa}$ \\
\hline SEGli & $7,3 \pm 0,3 \mathrm{Aa}$ & $6,7 \pm 0,3 \mathrm{Aa}$ & $6,8 \pm 0,3 \mathrm{Aa}$ & $7,0 \pm 0,2 \mathrm{Aa}$ & $7,3 \pm 0,4 \mathrm{Aa}$ \\
\hline SEMalt & $7,2 \pm 0,4 \mathrm{Aa}$ & $6,8 \pm 0,3 \mathrm{Aa}$ & $6,8 \pm 0,3 \mathrm{Aa}$ & $6,9 \pm 0,3 \mathrm{Aa}$ & $7,2 \pm 0,2 \mathrm{Aa}$ \\
\hline \multirow[t]{2}{*}{ SEMg } & $7,1 \pm 0,1 \mathrm{ABa}$ & $6,7 \pm 0,1 \mathrm{Ba}$ & $6,8 \pm 0,2 \mathrm{Ba}$ & $7,0 \pm 0,4 \mathrm{ABa}$ & $7,3 \pm 0,2 \mathrm{ABa}$ \\
\hline & - & Sódio $\mathrm{mmol} \mathrm{L}^{-1}$ (n & \pm desvio padrão) -- & - & ---------------- \\
\hline SE & $140,7 \pm 4,5 \mathrm{ABa}$ & $140,7 \pm 2,4 \mathrm{ABa}$ & $146,2 \pm 4,2 \mathrm{Aa}$ & $140,0 \pm 3,2 \mathrm{ABa}$ & $134,2 \pm 1,7 \mathrm{Bb}$ \\
\hline SEGli & $142,2 \pm 3,9$ Аа & $142,5 \pm 3,9 \mathrm{Aa}$ & $142,0 \pm 4,7 \mathrm{Aa}$ & $140,5 \pm 5,3 \mathrm{Aa}$ & $137,0 \pm 0,8 \mathrm{Aab}$ \\
\hline SEMalt & $141,0 \pm 5,3 \mathrm{Aa}$ & $141,2 \pm 2,2 \mathrm{Aa}$ & $144,5 \pm 8,2 \mathrm{Aa}$ & $140,0 \pm 1,9 \mathrm{Aa}$ & $140,2 \pm 4,4 \mathrm{Aa}$ \\
\hline \multirow{2}{*}{ SEMg } & $137,5 \pm 1,7 \mathrm{ABa}$ & $142,5 \pm 3,9 \mathrm{Aa}$ & $143,5 \pm 4,0 \mathrm{Aa}$ & $138,7 \pm 3,0 \mathrm{ABa}$ & $135,5 \pm 1,7 \mathrm{Bab}$ \\
\hline & $-\cdots$ & otássio mmol L ${ }^{-1}$ & a \pm desvio padrão) & (-) & ------------------- \\
\hline $\mathrm{SE}$ & $4,25 \pm 0,25 \mathrm{Aa}$ & $3,52 \pm 0,64 \mathrm{Aa}$ & $3,67 \pm 0,24 \mathrm{Aa}$ & $3,65 \pm 0,44 \mathrm{Aa}$ & $4,05 \pm 0,24 \mathrm{Aa}$ \\
\hline SEGli & $4,12 \pm 0,17 \mathrm{Aa}$ & $3,22 \pm 0,27 \mathrm{Aa}$ & $3,27 \pm 0,29$ Aа & $3,72 \pm 0,83 \mathrm{Aa}$ & $4,05 \pm 0,42 \mathrm{Aa}$ \\
\hline SEMalt & $4,22 \pm 0,13 \mathrm{Aa}$ & $3,02 \pm 0,96 \mathrm{Ba}$ & $3,27 \pm 0,57 \mathrm{ABa}$ & $3,50 \pm 0,29 \mathrm{ABa}$ & $3,57 \pm 0,86 \mathrm{ABa}$ \\
\hline \multirow[t]{2}{*}{ SEMg } & $4,10 \pm 0,37 \mathrm{Aa}$ & $3,60 \pm 0,27 \mathrm{Aa}$ & $3,45 \pm 0,49 \mathrm{Aa}$ & $3,67 \pm 0,56$ Аа & 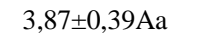 \\
\hline & 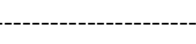 & Cloreto mmol L ${ }^{-1}$ & adesvio padrão) - & & \\
\hline SE & $106,5 \pm 2,6 \mathrm{ABa}$ & $106,0 \pm 2,8 \mathrm{ABa}$ & $109,7 \pm 4,2 \mathrm{Aa}$ & $104,5 \pm 2,9 \mathrm{ABa}$ & $101,2 \pm 2,2 \mathrm{Ba}$ \\
\hline SEGli & $108,5 \pm 2,6$ Aа & $108,5 \pm 4,5 \mathrm{Aa}$ & $105,5 \pm 3,5 \mathrm{Aa}$ & $105,7 \pm 4,1 \mathrm{Aa}$ & $103,5 \pm 1,7 \mathrm{Aa}$ \\
\hline SEMalt & $108,0 \pm 4,2 \mathrm{Aa}$ & $106,5 \pm 1,7 \mathrm{Aa}$ & $107,0 \pm 5,0$ Aа & $102,5 \pm 0,6$ Aa & $103,2 \pm 2,6$ Aa \\
\hline \multirow[t]{2}{*}{ SEMg } & $105,5 \pm 1,7 \mathrm{ABa}$ & $108,5 \pm 1,0 \mathrm{Aa}$ & $108,0 \pm 2,6 \mathrm{Aa}$ & $104,5 \pm 3,3 \mathrm{ABa}$ & $101,7 \pm 1,0 \mathrm{Ba}$ \\
\hline & & & édia \pm desvio padr & $205+021 \mathrm{AB}$ & 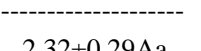 \\
\hline SE & 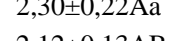 & 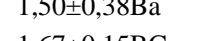 & $1,80 \pm 0,22 \mathrm{ABa}$ & 2,05 $\pm 0,21 \mathrm{ABa}$ & 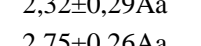 \\
\hline SEGli & $2,12 \pm 0,13 \mathrm{ABa}$ & 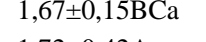 & $1,52 \pm 0,35 \mathrm{Ca}$ & $2,42 \pm 0,22 \mathrm{Aa}$ & $2,75 \pm 0,26 \mathrm{Aa}$ \\
\hline SEMalt & $2,42 \pm 0,52 \mathrm{Aa}$ & $1,72 \pm 0,42 \mathrm{Aa}$ & $1,60 \pm 0,42 \mathrm{Aa}$ & $2,15 \pm 0,24 \mathrm{Aa}$ & $2,47 \pm 0,36$ Аа \\
\hline \multirow[t]{2}{*}{ SEMg } & $2,10 \pm 0,14 \mathrm{Aa}$ & $2,17 \pm 0,30 \mathrm{Aa}$ & $2,02 \pm 0,22 \mathrm{Aa}$ & $2,10 \pm 0,18 \mathrm{Aa}$ & $2,32 \pm 0,15 \mathrm{Aa}$ \\
\hline & - & cio iônico mmol L & dia \pm desvio padrã & 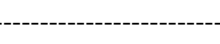 & --------------------- \\
\hline SE & $1,78 \pm 0,04 \mathrm{Aa}$ & $1,63 \pm 0,19$ Аа & $1,79 \pm 0,10 \mathrm{Aa}$ & $1,87 \pm 0,09 \mathrm{Aa}$ & $1,78 \pm 0,10 \mathrm{Aa}$ \\
\hline SEGli & $1,85 \pm 0,10 \mathrm{Aa}$ & $1,70 \pm 0,13 \mathrm{Aa}$ & $1,57 \pm 0,29 \mathrm{Aa}$ & $1,85 \pm 0,05 \mathrm{Aa}$ & $1,80 \pm 0,04 \mathrm{Aa}$ \\
\hline SEMalt & $1,82 \pm 0,05 \mathrm{Aa}$ & $1,55 \pm 0,08 \mathrm{Aa}$ & $1,53 \pm 0,27 \mathrm{Aa}$ & $1,78 \pm 0,26 \mathrm{Aa}$ & $1,81 \pm 0,14 \mathrm{Aa}$ \\
\hline SEMg & $1,72 \pm 0,06 \mathrm{Aa}$ & $1,77 \pm 0,06 \mathrm{Aa}$ & $1,70 \pm 0,14 \mathrm{Aa}$ & $1,84 \pm 0,08 \mathrm{Aa}$ & $1,78 \pm 0,07 \mathrm{Aa}$ \\
\hline SE & $10,79 \pm 0,95 \mathrm{Bab}$ & $13,07 \pm 1,88 \mathrm{Ba}$ & $16,07 \pm 1,35 \mathrm{Aa}$ & $13,04 \pm 0,34 \mathrm{Ba}$ & $12,15 \pm 0,46 \mathrm{Ba}$ \\
\hline SEGli & $8,64 \pm 1,33 \mathrm{Bb}$ & $10,26 \pm 1,43 \mathrm{ABa}$ & $12,02 \pm 0,84 \mathrm{Ab}$ & $10,84 \pm 0,56 \mathrm{ABb}$ & $9,24 \pm 0,84 \mathrm{Bb}$ \\
\hline SEMalt & $10,00 \pm 0,87 \mathrm{Bab}$ & $12,89 \pm 1,69 \mathrm{ABa}$ & $14,96 \pm 2,43 \mathrm{Aab}$ & $12,72 \pm 1,73 \mathrm{ABab}$ & $12,15 \pm 1,88 \mathrm{ABa}$ \\
\hline SEMg & $12,43 \pm 1,64 \mathrm{Ba}$ & $13,83 \pm 1,96 \mathrm{ABa}$ & $15,95 \pm 2,00 \mathrm{Aa}$ & $13,00 \pm 0,68 \mathrm{ABa}$ & $12,32 \pm 0,64 \mathrm{Ba}$ \\
\hline
\end{tabular}

Análise de variância (medidas repetidas)

As médias na mesma coluna seguidas por letras minúsculas diferentes e as médias na mesma linha seguidas por letras maiúsculas diferentes diferem pelo teste de Turkey $(\mathrm{P}<0,05)$.

T0h (imediatamente antes do início da hidratação), T6h (seis horas de hidratação), T12h (12 horas de hidratação), T18h (seis horas após o término da hidratação) e T24h (12 horas após o término da hidratação)

Grupo SE - a solução foi preparada utilizando-se 5,27g de $\mathrm{NaCl}$, 0,37g de $\mathrm{KCl}$ e 3,78g de $\mathrm{NaHCO}_{3}$ em 1.000 mL de água (osmolaridade calculada: 280mosmol L ${ }^{-1}$ ); grupo SEGli - a solução foi preparada adicionando 10,8g de glicose em um litro da solução eletrolítica (osmolaridade calculada: 340mosmol L ${ }^{-1}$ ); grupo SEMalt - a solução foi preparada adicionando 18g de maltodextrina em um litro da solução eletrolítica (osmolaridade calculada: 290mosmol $\mathrm{L}^{-1}$ ) e grupo SEMg - a administração da solução eletrolítica foi precedida pela administração de uma dose de sulfato de magnésio $\left(1 \mathrm{~g} \mathrm{~kg}^{-1}\right)$ diluído nos primeiros cinco litros da solução eletrolítica. 
Na tabela 1, pode-se constatar que os valores do potássio plasmático no E1Des não apresentaram diferença $(\mathrm{P}>0,05)$ em todos os tratamentos ao longo de toda fase experimental. Além disso, a comparação entre tratamentos mostrou diminuição $(\mathrm{P}<0,05)$ nos valores do potássio plasmático, nos animais do SEMalt no T6 (metade do período de hidratação), dado também sem significado clínico, pois o valor obtido $\left(3,22 \pm 0,27 \mathrm{mmol} \mathrm{L}^{-1}\right)$ também está na faixa de referência descrita por KANEKO et al. (1997) e RADOSTITS et al. (2000). Os animais do E2Hig não apresentaram diferença significativa entre os tratamentos $(\mathrm{P}>0,05)$ e nos tramentos ao longo da fase experimental (Tabela 2), com exceção dos animais do SEMalt na sexta hora de hidratação (T6h). Apesar dessa discreta diminuição, os valores continuaram na faixa de normalidade para a espécie equina (KANEKO et al., 1997).

O teor de cloreto (Tabelas 1 e 2) nos E1Des e E2Hig não apresentou diferença significativa entre os tratamentos $(\mathrm{P}>0,05)$. Entretanto, no E1Des, os maiores valores do cloreto plasmático $(\mathrm{P}<0,05)$ foram detectados na sexta hora de hidratação (T6h), em todos os tratamentos. A possível causa desse aumento foi a formulação das soluções utilizadas, que, apesar de conterem 95,06mmol de cloreto por litro de solução, valores semelhantes aos do plasma, ocasionou pequeno aumento nos valores desse íon, sinalizando que possivelmente soluções contendo menores concentrações de cloreto sejam mais adequadas. Mesmo assim os valores obtidos permaneceram na faixa de normalidade (KANEKO et al., 1997; RADOSTITS et al., 2000). Por sua vez, no E2Hig, os tratamentos SE (T12h) e SEMg (T6h e T12h) apresentaram aumento $(\mathrm{P}<0,05)$ nos índices do cloreto. Possivelmente a causa para esse evento tenha sido a mesma descrita para os animais do E1Des. Os menores índices $(\mathrm{P}<0,05)$ foram observados no T24 (final do período de observação) em consequência do término da hidratação e ingestão espontânea de água pelos animais.

No presente estudo, a concentração do magnésio total (tMg) nos animais do E1Des apresentou diferença $(\mathrm{P}<0,05)$ entre tratamentos no T6 (Tabela 1$)$. Os maiores valores foram registrados nos animais que receberam SEMg. A avaliação dos tratamentos ao longo do tempo (T0h a T24h) mostrou os menores índices $(\mathrm{P}<0,05)$ de $\mathrm{tMg}$ no T12h. Com exceção do grupo SEMg no $\mathrm{T} 6$, os resultados eram esperados, pois o que se buscava com o uso dessa solução (SEMg) era uma ação mínima sobre a absorção de água e magnésio pelo trato gastrintestinal e máximo efeito na hidratação da ingesta. Esses resultados demonstram que uma pequena parte do magnésio contido no $\mathrm{MgSO}_{4}$ é absorvida. O magnésio total (tMg) não apresentou diferença entre tratamentos $(\mathrm{P}>0,05)$ no experimento $2 \mathrm{H}$ (Tabela 2). A concentração plasmática de tMg diminuiu $(\mathrm{P}<0,05)$ nos tratamentos SE (T6) e SEGli (T12). Nos animais que receberam a SEMalt, houve também diminuição nos valores do tMg em T6 e T12, entretanto a mesma não foi significativa $(\mathrm{P}>0,05)$. Esse resultado pode ter sido determinado pela ausência de magnésio nessas soluções, o que está de acordo com DIPALMA (1990), DART et al. (1992), OLERICH \& RUDE (1994)e ALVES et al. (2005), os quais relatam a ocorrência de hipomagnesemia após hidratação prolongada com soluções sem magnésio. Esse achado acena para a necessidade da adição do magnésio em soluções eletrolíticas de manutenção, principalmente se o animal for acometido por obstrução simples do intestino grosso (COSTA et al., 2002), afeç̧ões estrangulantes e íleo paralítico (GARCIA-LOPEZ et al., 2001), pois usualmente esses processos desencadeiam hipomagnesemia. Por sua vez, os animais tratados com a solução SEMg apresentaram valores de magnésio total constantes durante toda a fase experimental.

O cálcio iônico (iCa) reflete mais acuradamente o status do cálcio no organismo. Diminuição na concentração de cálcio contribui para o desenvolvimento do íleo intestinal em equinos (GARCIA-LOPEZ., 2001). Esse fato sugere que as soluções eletrolíticas administradas por via enteral ou parenteral devem conter cálcio na sua composição, principalmente nos casos de cólica e diarréia em animais dessa espécie. No presente estudo, conforme apresentado nas tabelas 1 e 2, os E1Des e E2Hig não apresentaram diferença significativa entre os tratamentos $(\mathrm{P}>0,05)$. O cálcio iônico no E1Des apresentou diminuição $(\mathrm{P}<0,05)$ apenas no grupo SEMalt, na fase final da hidratação (T12). Mesmo assim, os valores obtidos $\left(1,47 \pm 0,10 \mathrm{mmol} \mathrm{L}^{-1}\right)$ permaneceram na faixa de normalidade (1,3 a 1,5mmol $\left.\mathrm{L}^{-1}\right)$, segundo DART et al., (1992). Por sua vez, nos animais do E2Hig, não ocorreu diferença $(\mathrm{P}>0,05)$ durante toda a fase experimental.

A avaliação ao longo da fase experimental, em E1Des e E2Hig, demonstrou o maior volume plasmático $(\mathrm{P}<0,05)$ em todos os tratamentos no T12 (final da fase de hidratação), indicando que a administração da HET foi eficiente em expandir o volume plasmático. No E1Des, não foi detectada diferença entre tratamentos $(\mathrm{P}>0,05)$, ocorrendo diferença $(\mathrm{P}<0,05)$ nos tratamentos ao longo do tempo (Tabela 1). No E2Hig, foi observada diferença $(\mathrm{P}<0,05)$ entre tratamentos (Tabela 2). No T6h e T12h, foram constatados os maiores índices de volume plasmático. Porém, no T12h (final da fase de hidratação), os animais que receberam 
solução contendo glicose (SEGli) apresentaram a menor expansão plasmática. Esse efeito provavelmente se deveu à osmolaridade da SEGli $\left(340 \mathrm{mmOsm} \mathrm{L}^{-1}\right)$, que reduziu a absorção da solução eletrolítica, determinando a menor expansão do volume plasmático. Além disso, os resultados observados no presente estudo possivelmente foram também ocasionados por particularidades do trato gastrintestinal dos eqüinos, como, por exemplo, o trânsito rápido de fluidos pelo intestino delgado, que talvez tenha também contribuído para os resultados obtidos. Em outras espécies, a adição de glicose à solução para hidratação enteral maximiza a absorção de água através do mecanismo de absorção combinada de glicose e sódio pela mucosa do intestino delgado(GISOLFI\& DUCHMAN, 1992; CONVERTINO et al., 1996). Entretanto, resultados semelhantes aos do presente ensaio foram obtidos por SOSA LEON et al. (1995) e MONREAL et al. (1999) também na espécie equina com hidratação enteral com soluções eletrolíticas contendo glicose. Nos animais que receberam SEMg, foram detectados os maiores valores de volume plasmático $(\mathrm{P}<0,05)$. Esse achado foi inesperado, pois a dose utilizada de sulfato de magnésio $\left(1 \mathrm{~g} \mathrm{~kg}^{-1}\right)$ tem efeito laxativo (WHITE \& DABARAINER, 1997), por atrair e reter água no lume intestinal (GOODMAN \& GILMAN, 1996), conseqüentemente esperava-se algum grau de efeito sobre o volume plasmático.

\section{CONCLUSÕES}

A fluidoterapia enteral administrada por sonda nasoesofágica de forma contínua no volume de $15 \mathrm{~mL} \mathrm{~kg}^{-1} 12 \mathrm{~h}^{-1}$, em ambos os experimentos, promoveu a expansão do volume plasmático, diminuiu os valores de PPT e do hematócrito e manteve as concentrações de sódio, potássio, cloreto e cálcio iônico dentro da faixa de normalidade. A solução eletrolítica contendo sulfato de magnésio teve efeito na manutenção da homeostase do magnésio plasmático. A adição de glicose e da maltodextrina à solução eletrolítica não ocasionou expansão satisfatória do volume plasmático.

\section{FONTES DE AQUISIÇÃO}

a - Veterinary Enteral Feeding Tube NG 1450 - Medical Instrumentation for Animals, EUA.

b - Maltodextrina - Arve industria e comércio Ltda. - Centev/ UFV, Viçosa - MG.

c - Vacuntainer BD - Becton \& Dickinson ind. Cirúrgica Ltda., Brasil.

d - Fotômetro de chama B462 - Micronal

e - AIRONE 200 - Winner - ARG

f - AVL 9180 - Roche

g - ELISA - PR 2100 Reader da SANOFI, França.

\section{COMITÊ DE ÉTICA E BIOSSEGURANÇA}

Foi submetido à Comissão de Ética e Biossegurança do DVT/UFV. Protocolo 26/2006.

\section{REFERÊNCIAS}

ALVES, G.E.S. et al. Tratamento da compactação experimental do cólon maior em eqüinos: resultados de laboratório e exames bioquímicos. Arq Bras Med Vet Zootec, v.57, p.281-287, 2005. Disponível em: http://www.scielo.br/scielo.php?script=sci_arttext\&pid=S010209352005000300001\&lng=en\&nrm=iso\&tlng=pt. Doi: 10.1590/ S0102-09352005000300001.

AVANZA, M.F.B. Solução eletrolítica associada ou não a glicose, maltodextrina e sulfato de magnésio administrada por via enteral em eqüinos hígidos e desidratados experimentalmente. 2007. 76f. Dissertação (Mestrado em Medicina Veterinária) - Curso de Pós-graduação em Medicina Veterinária, Universidade Federal de Viçosa.

AVANZA, M.F.B. et al. Fluidoterapia em vacas normais e experimentalmente desidratadas. In: COMBRAVET, 2004, São Luís, MA. Anais... São Luís: Universitária, 2004. p.31.

CONVERTINO, V.A. et al. American College of Sports Medicine position. Exercise e fluid replacement. Medicine Science Sports Exercise, v.28, p.i-vii, 1996.

COSTA, L.R.R. et al. Plasma magnesium and other electrolyte abnormalities in horses affected with gastrointestinal tract disease. In: INTERNATIONAL COLIC RESEARCH SYMPOSIUM, 2002, Manchester, UK. Proceedings... Manchester: Manchester Comference Center, 2002. p.95-96.

DART, AJ. et al. Ionized calcium concentration in horses with surgically managed gastrointestinal disease: 147 cases (19881990). J Am Vet Med Assoc, v.191, p.849-854, 1992.

DiPALMA, JR. Magnesium replacement therapy. American Family Physician, v.42, p.173-176, 1990.

GARCIA-LOPEZ, JM. et al. Prevalence and prognostic importance of hypomagnesaemia in horses that have colic surgery. Am J Vet Res, v.62, p.7-12, 2001.

GISOLFI, C.V.; DUCHMAN, S.M. Guidelines for optimal replacement beverages for different athletic events. Medicine Science Sports Exercise, v.24, p.679-687, 1992.

GOODMAN, L.S.; GILMAN, A. As bases farmacológicas da terapêutica. 8.ed. Rio de Janeiro: McGran Hill, 1996. 1821 p.

JAIN, N.C. Veterinary hematology. 4.ed. Philadelphia: Lea \& Febier, 1986. 1221p.

KANEKO, J.R. et al. Clinical biochemistry of domestic animal. 5.ed. San Diego: Academic, 1997. 932p.

LOPES, M.A.F. et al. Treatments to promote colonic hydration: enteral fluid therapy versus intravenous fluid therapy e magnesium sulphate. Equine Vet J, v.34, p.505-509, 2002.

MONREAL, L. et al. Electrolyte vs. glucose-electrolyte isotonic solutions for oral rehydration therapy in horses. Equine Vet J, Suppl, v.30 p.425-429, 1999.

Ciência Rural, v.39, n.4, jul, 2009. 
OLERICH, MA.; RUDE, RK. Should we supplement magnesium in critically ill patients. New Horizons, v.2, p.186-192, 1994.

RADOSTITS, O.M. et al. Diseases of the no-ruminat stomach and intestines. In: ______. Veterinary medicine: a textbook of the diseases of cattle, sheep, pigs, goats and horses. 9.ed. London: Saunders, 2000. p.197-246.

RIBEIRO FILHO, J.D.; ALVES, G.E.S. Compactação do intestino grosso de eqüinos: estudo comparativo de três protocolos de indução. Arq Bras Med Vet Zootec, v.54, p.370-375, 2002. Disponível em: http://www.scielo.br/scielo.php?script=sci_arttext\&pid=S010209352002000400007\&lng=en\&nrm=iso\&tlng=pt. Doi: 10.1590/ S0102-09352002000400007.

RIBEIRO FILHO, J.D. et al. Hemogasometria em eqüinos com compactação experimental do cólon maior tratados com sene, fluidoterapia enteral e parenteral. Ciênc Rural, v.37, p.755-761, 2007. Disponível em: http://www.scielo.br/ scielo.php? script=sci_art text \& pid=S0103-
$84782007000300024 \& \operatorname{lng}=$ en $\& n r m=$ iso\&tlng=pt. Doi: 10.1590/S0103-84782007000300024.

RIBEIRO FILHO, J.D. et al. Tratamento de bovinos desidratados com fluidoterapia via sonda nasogástrica de pequeno calibre. In: COMBRAVET, 2004, São Luís, MA. Anais... São Luís: Universitária, 2004. p.33.

SAEG - Sistema para análises estatísticas, Versão 9.1. Viçosa: UFV - Fundação Arthur Bernardes, 2007. 301p.

SEAHORN, T.L.; CORNICK-SEARHORN, J. Fluid therapy. Vet Clin North Am Equine Pract, v.10, p.517-525, 1994.

SOSA LEON, L.A. et al. The effects of tonicity, glucose concentration e temperature of an oral rehydration solution on its absorption e elimination. Equine Vet J, v.20, Suppl, p.140-146, 1995.

WHITE, N.A.; DABAREINER, R.M. Treatment of impaction colics. Vet Clin North Am Equine Pract, v.13, p.243-259, 1997. 